\title{
FINAL GRANT REPORT TO THE DEPARTMENT OF ENERGY Shock Stability in Systems that Change Type
}

PI Name: Barbara Lee Keyfitz

PI Institution: University of Houston

PI Address: Houston, Texas 77204-3476

\author{
Award Number: DE-FG05-91ER25102 \\ Report for Period: FINAL \\ Date of Report: November 10, 1995
}

\section{Aims}

The aim of the original project was to investigate systems of conservation laws that change type. Progress was made on this problem. During the last period of the grant, the PI began an investigation of a multidimensional system related to Mach reflection which goes beyond the original work proposed. This has been fruitful direction in which to apply expertise on change of type. Some basic theoretical results have been found.

\section{Results}

One focus of the research has been to understand where unsteady equations that change type come from in models. We have made some progress: publication [4], below, showed that systems in which strict hyperbolicity fails along a curve in phase space occur frequently in models for multiphase saturation equations. In addition, perturbations of these equations typically exhibit elliptic regions.

We also began an investigation of the relation between inaccessible regions in change-oftype models and in systems where genuine nonlinearity fails (described in the same paper): there are resemblences between the phenomena observed in the two types of models. A characterization of these phenomena in multiphase flow models will be very useful in applications, since engineers frequently encounter this situation and are confused about what the apparent ill-posedness implies for their models and computations involving them. The current results give a partial answer: the models may be ill-posed on a sufficiently small scale that it is not significant for the application; furthermore, the solutions may not be unstable (again, depending on the scale), and resemble the solutions of strictly hyperbolic systems to which they are related by formal perturbation. These results are reported in the survey paper, [7].

A related problem is currently part of the dissertation research of a PhD student, Zhuang Zhi Zhang. Following work of Vaidyanath Vinod, who received his degree in 1992, and worked on perturbations of a quadratic nonstrictly hyperbolic system, Zhang is looking at a nonstrictly hyperbolic system with the interesting behavior of allowing overlapping rarefaction waves.

In joint work with Milton Lopes, of Campinas University in Brazil, an existence theorem was proved for nonuniform solutions of a system with elliptic and hyperbolic regions, [5]. Here we were able to show that if a shock between regions of different type satisfies the generalized geometric entropy condition, in which the standard Lax criterion relating 


\section{DISCLAIMER}

This report was prepared as an account of work sponsored by an agency of the United States Government. Neither the United States Government nor any agency thereof, nor any of their employees, make any warranty, express or implied, or assumes any legal liability or responsibility for the accuracy, completeness, or usefulness of any information, apparatus, product, or process disclosed, or represents that its use would not infringe privately owned rights. Reference herein to any specific commercial product, process, or service by trade name, trademark, manufacturer, or otherwise does not necessarily constitute or imply its endorsement, recommendation, or favoring by the United States Government or any agency thereof. The views and opinions of authors expressed herein do not necessarily state or reflect those of the United States Government or any agency thereof. 


\section{DISCLAIMER}

Portions of this document may be illegible in electronic image products. Images are produced from the best available original document. 
shock and characteristic speeds is replaced by one relating the shock to the real part of the characteristic speed, then perturbing the Cauchy data results in existence, for a finite but uniform time, of a smooth flow on both sides of a smoothly perturbed shock. The perturbed Cauchy data are required to be in a special class of uniformly analytic functions, with which we were able to prove a uniform Cauchy-Kowalewski existence theorem in the elliptic region, and then match the solution to that in the hyperbolic region using the Rankine-Hugoniot relation to determine the shock as a free boundary. The result proved is for a system of two conservation laws, and makes extensive use of Riemann invariants.

In the paper [3], Keyfitz and Lopes made a study of properties of systems of conservation laws in several space variables which respect the Euclidean symmetry (rotation and reflection) of the spatial variables. We noted that the dependent variables could be either invariant under the group action, as is, for example, density, or equivariant pairs such as velocity fields. We found that only certain combinations of invariant and equivariant variables were compatible with a strictly hyperbolic structure, with nondegenerate wave cones, or with genuine nonlinearity. For example, there is no system of two equations which both respects Euclidean symmetry and has the strictly hyperbolic, genuinely nonlinear properties one would like for a prototype model; the simplest model (in two space variables) with all these properties is the nonlinear wave equation.

This research also explains why there is no satisfactory definition of genuine nonlinearity for a scalar equation in more than one space dimension: it is the nature of such an equation to reflect a degree of anisotropy.

The paper on singular shocks (publication [2], below), with Herbert Kranzer of Adelphi University, completes the project of finding a rigorous analytical description of singular shocks and their convergence in a model problem. We have also found some further properties and characterizations of these objects; with a slightly expanded definition, we can now describe nonuniform singular shocks.

In the most recent work, with Sunčica Čanić, at Iowa State University, we have formulated a theory for the transition from regular to Mach reflection in weak shocks. We have a description for a simplified model, the unsteady transonic small disturbance equation, which ignores entropy waves and is a valid description of the fluid dynamics only near the shock. Our idea is to analyze self-similar solutions to Riemann problems using a lower-dimensional analysis of the similarity equations. These equations behave like standard conservation laws in many ways; however, they are nonautonomous, and they change type in a way somewhat different both from steady transonic flow and from unsteady time-dependent problems in one space dimension. Furthermore, this degeneracy in the elliptic problem, when linearized, behaves differently from the familiar Tricomi problem, and has not previously been associated with transonic flow. We proved an existence theorem for this equation, [6]. We are now working on the free-boundary problem. Eventually, we hope to generalize to a larger class of equations. This will have exciting implications for analytical study of multidimensional conservation law problems, since the Mach reflection problem is merely a prototype of the possible complexity of solutions.

The project, now focussing on multidimensional Riemann problems, continues under Department of Energy Grant DE-FG03-94ER25222, titled 'Selfsimilar Solution of Multidimensional Conservation Laws'. With Čanić, I hope to continue work on the small-disturbance version of the Mach reflection problem, and to complete the analysis. 


\section{Publications Acknowledging the Grant}

Papers which have been accepted or have appleared are listed here. Future papers will acknowledge DOE Grant DE-FG03-94ER25222 which continues this project.

1. B. L. Keyfitz, 'Development of singularities in Riemann invariants', UH Math Department Research Report UH/MD-129, 1992. Research supported by the Texas Advanced Research Program under Grant 003652124ARP and the Department of Energy, grant DE-FG05-91ER25102, and NSF, grant DMS-91-03560.

2. B. L. Keyfitz and H. C. Kranzer, 'Spaces of weighted measures for conservation laws with singular shock solutions', Journal of Differential Equations, 118, (1995), 420-451. Research supported by NSF/AFOSR under grant number 89-03768; DOE under grant DE-FG05-91ER25102, NSF grant DMS-91-03560 and TARP grant 003652-0077.

3. B. L. Keyfitz and M. Lopes, 'How to use symmetry to find models for multidimensional conservation laws', (UH Math Department Research Report, November 1992); Proceedings of AMS/STAM Summer Seminar on Exploiting Symmetry in Applied and Numerical Analysis (G. Allgower, K. Georg and R. Miranda, eds), AMS, Lectures in Applied Mathematics, 29 (1993), 273-284. Research supported by the Texas Advanced Research Program under Grant 00365-2124-ARP, and the Department of Energy, grant DE-FG05-91ER25102, and NSF, grant DMS-91-03560.

4. B. L. Keyfitz, 'Multiphase saturation equations, change of type and inaccessible regions', (UH Math Department Research Report UH/MD-155, 1992); Proceedings of the 1992 Oberwolfach Conference on Porous Media (J. Douglas and U. Hornung, eds), Birkhäuser, Int. Ser. of Num. Math, 114, 103-116. Research supported by the Texas Advanced Research Program under Grant 00365-2124-ARP, and the Department of Energy, grant DE-FG05-91ER25102, and NSF, grant DMS-91-03560, with support from AFOSR.

5. B. L. Keyfitz and M. Lopes, 'A geometric study of shocks in equations that change type', Journal of Dynamics and Differential Equations, 6 (1994), 351-393. Research supported by the Texas Advanced Research Program under Grant 00365-2124-ARP and the Department of Energy, grant DE-FG05-91ER25102, and NSF, grant DMS-9103560 .

6. S. Canic and B. L. Keyfitz, 'An Elliptic Problem Arising from the Unsteady Transonic Small Disturbance Equation', to appear in JDE. Research supported by the Texas Advanced Research Program under Grant 00365-2124-ARP and the Department of Energy, grant DE-FG05-91ER25102, and NSF, grant DMS-91-03560.

7. B. L. Keyfitz, 'A Geometric Theory of Conservation Laws which Change Type', Zeitschrift für Angewandte Mathematik und Mechanik, 75, (1995), 571-581. Research supported by the Texas Advanced Research Program under Grant 00365-2102-ARP and the Department of Energy, grant DE-FG05-91ER25102. 